INPLASY

PROTOCOL

To cite: Choi et al. The effectiveness and safety of acupuncture combination treatment on smoking cessation: A protocol for systematic review and/or meta-analysis. Inplasy protocol 202230023. doi: 10.37766/inplasy2022.3.0023

Received: 05 March 2022

Published: 05 March 2022

Corresponding author:

Won-Suk Sung

1984sws@hanmail.net

Author Affiliation:

Dongguk University Bundang Oriental Hospital.

Support: None.

Review Stage at time of this submission: The review has not yet started.

Conflicts of interest: None declared.

\section{The effectiveness and safety of acupuncture combination treatment on smoking cessation: A protocol for systematic review and/or meta-analysis}

Choi, IS1; Kim, EJ2; Seo, BK³; Kim, GW4.

Review question / Objective: The aim of this systematic review (SR) and meta-analysis is to investigate the clinical effectiveness and safety of combined acupuncture treatment for smoking cessation.

Condition being studied: A clinical trial compared the smoking cessation effect of auricular acupuncture, nicotine patch, and combination treatment and reported that combination treatment group decreased the degree of nicotine dependence significantly than other monotherapy groups. Information sources: A search will be conducted from inception to July 2022 in the following databases: MEDLINE, Cochrane Library, China National Knowledge Infrastructure (CNKI), CiNii, J-STAGE, KoreaMed, Korean Medical Database, Korean Studies Information Service System (KISS), National Digital Science Library (NDSL), Korea Institute of Science and Technology Information (KISTI), and Oriental Medicine Advanced Searching Integrated System (OASIS).

INPLASY registration number: This protocol was registered with the International Platform of Registered Systematic Review and Meta-Analysis Protocols (INPLASY) on 05 March 2022 and was last updated on 05 March 2022 (registration number INPLASY202230023).

\section{INTRODUCTION}

Review question / Objective: The aim of this systematic review (SR) and metaanalysis is to investigate the clinical effectiveness and safety of combined acupuncture treatment for smoking cessation.
Rationale: Acupuncture has been known to be popular and safe treatments for smoking cessation. There are several SRs about acupuncture monotherapy, but there has been no SR about acupuncture combination treatment comparing other conventional monotherapy. 
Condition being studied: A clinical trial compared the smoking cessation effect of auricular acupuncture, nicotine patch, and combination treatment and reported that combination treatment group decreased the degree of nicotine dependence significantly than other monotherapy groups.

\section{METHODS}

Search strategy: We will search the studies about the acupuncture combined treatment for smoking cessation through multiple electronic databases, manual search, and contact to author.

Participant or population: Tobacco smokers who wished to stop smoking.

Intervention: Acupuncture with conventional treatment for smoking cessation.

Comparator: Participants can undergo any type of conventional treatment for smoking cessation including nicotine replacement and counseling.

Study designs to be included: We will include randomized controlled trials (RCTs). If the number of included RCTs is insufficient, non-RCTs such as pilot studies could be included and we will describe it.

Eligibility criteria: The eligible interventions include any type of acupuncture including body acupuncture, ear acupuncture, auricular acupressure, and acupuncture with other type of acupuncture.

Information sources: A search will be conducted from inception to July 2022 in the following databases: MEDLINE, Cochrane Library, China National Knowledge Infrastructure (CNKI), CiNii, JSTAGE, KoreaMed, Korean Medical Database, Korean Studies Information Service System (KISS), National Digital Science Library (NDSL), Korea Institute of Science and Technology Information (KISTI), and Oriental Medicine Advanced Searching Integrated System (OASIS).
Main outcome(s): Abstinence rate will be the primary outcome. Abstinence rates can be measured by the change of cigarette consumption, cigarette withdrawal symptom scores, or self-developed criteria.

Additional outcome(s): Adverse events and any biochemical indicator.

Quality assessment / Risk of bias analysis: Two reviewers will assess the risk of bias independently by using risk of bias (ROB) tool from the Cochrane Collaboration. The tool is composed of seven domains: sequence generation, allocation concealment, blinding of participants and investigators, blinding of outcome assessors, incomplete outcome data, selective outcome reporting, and other biases. The risk of bias will be rated as "low risk," "high risk," or "unclear risk" for each domain.

Strategy of data synthesis: We will perform a meta-analysis by using the Review Manager (RevMan) software for Windows (Version 5.3; Copenhagen; The Nordic Cochrane Center, The Cochrane Collaboration, 2014). We will combine the changes between baseline and intervention completion in the included studies. The mean difference (same outcome) or standardized mean difference (different outcome) and $95 \%$ confidence intervals will be calculated with random-effects model or a fixed-effect model.

Subgroup analysis: Potentially if data suitable.

Sensitivity analysis: We will perform a sensitivity analysis to test the robustness of study finding.

Country(ies) involved: Republic of Korea.

Keywords: smoking cessation, acupuncture combination treatment, systematic review, meta-analysis.

Contributions of each author:

Author 1 - In Suh Choi.

Email: reme2020@naver.com 
Author 2 - Eun-Jung Kim.

Email: hanijjung@naver.com

Author 3 - Byung-Kwan Seo.

Email: seohbk@hanmail.net

Author 4 - Geun-Woo Kim.

Email: kgwoo86@dongguk.ac.kr 